\title{
An integrated technological approach to the selection of lactic acid bacteria of flour origin for sourdough production
}

\author{
Luca Settanni *, Giusi Ventimiglia, Antonio Alfonzo, Onofrio Corona, Alessandro Miceli, Giancarlo Moschetti \\ Department of Agricultural and Forest Science, Università degli Studi di Palermo, Viale delle Scienze 4, 90128 Palermo, Italy
}

\section{A R T I C L E I N F O}

\section{Article history:}

Received 30 May 2013

Accepted 8 October 2013

Available online 16 October 2013

\section{Keywords:}

Fermentation

Lactic acid bacteria

Sourdough

Strain selection

Volatile organic compounds

Wheat flour

\begin{abstract}
A B S T R A C T
Several lactic acid bacteria (LAB) were evaluated in situ for their potential in sourdough fermentation. The strains belonged to Lactobacillus plantarum, Lactobacillus sakei, Lactobacillus sanfranciscensis, Leuconostoc citreum, Leuconostoc mesenteroides, Leuconostoc pseudomesenteroides and Weissella cibaria. LAB were used, in individual inocula, to carry out the fermentation of $\gamma$-ray treated (sterile) flour and untreated commercial flour, in order to evaluate their performances both in the absence and presence of the native microbiota of flour. The $\mathrm{pH}$ and total titratable acidity (TTA) showed a strong and fast acidification of the experimental sourdough determined by $W$. cibaria and $L n$. citreum strains. All strains were followed during fermentation by plate count. Randomly amplified polymorphic DNA (RAPD)-PCR analysis applied on the colonies isolated from the highest dilution of samples confirmed the dominance of the added strains in all sourdoughs prepared with sterile and non-sterile flour. The analysis of organic acids, performed by high-performance liquid chromatography (HPLC), confirmed that some $W$. cibaria and $L$. citreum strains showed an optimal fermentation quotient. The volatile organic compound (VOC) composition resulting from the gas chromatography coupled with mass spectrometry (GC/MS) analysis of sourdough headspace recognised 51 chemical compounds including acids, alcohols, aldehydes, esters, ketones, lactones, acetate, alkane, and phenol, most of which are of LAB origin and are relevant for the final bread. After baking, the breads were evaluated for the height of the central slices, colour of crust and crumb, hardness and number and distribution of alveolus. The combination of these results indicated that strains Ln. citreum PON10079 and PON10080 and W. cibaria PON10030 and PON10032 are suitable cultures to use in industrial production.
\end{abstract}

@ 2013 Elsevier Ltd. All rights reserved.

\section{Introduction}

The traditional biotechnology for the production of bread and other leavened baked products is based on sourdough, which is a complex microbial ecosystem mainly constituted by lactic acid bacteria (LAB) and yeasts (Vogel et al., 1999). Although LAB are primarily involved in the development of the sensory characteristics of sourdough, several by-products generated by yeasts during fermentation contribute to the improvement of the organoleptic complexity of the resulting doughs (Valmorri, Tofalo, Settanni, Corsetti, \& Suzzi, 2010). Thus, a successful sourdough fermentation is pivotal to providing the final products with the right attributes.

The typical LAB responsible for the acid production in sourdoughs belong to the genus Lactobacillus (Corsetti \& Settanni, 2007). Sourdough lactobacilli include obligately and facultatively heterofermentative and obligately homofermentative species (Hammes \& Vogel, 1995), but, contrary to the majority of other fermented food productions, the most defining role of these $\mathrm{LAB}$ in sourdough is played by the obligately

\footnotetext{
* Corresponding author. Tel.: + 39091 23896043; fax: + 390916515531.

E-mail address: luca.settanni@unipa.it (L. Settanni).
}

heterofermentive strains. This is due to the production of acetic acid in addition to the lactic acid from the fermentation of carbohydrates, important for the development of the right fermentation quotient (FQ = lactic/acetic acid molar ratio). Acetic acid strongly contributes to the aroma and the structure of the final products (Corsetti \& Settanni, 2007). Furthermore, heterofermentative LAB partly contribute to the process of dough leavening (Gobbetti et al., 1995).

Other species belonging to Enterococcus, Lactococcus, Leuconostoc, Pediococcus, Streptococcus and Weissella are commonly detected in sourdoughs (Corsetti \& Settanni, 2007), albeit at levels lower than those registered for lactobacilli. During the first stages of sourdough preparation, different non-Lactobacillus species have been found to prepare the environment for the establishment of the typical species (e.g. Lactobacillus sanfranciscensis) of mature sourdoughs (Corsetti, Settanni, Valmorri, Mastrangelo, \& Suzzi, 2007). These species are primarily sourced from cereals or flours (Alfonzo et al., 2013; Corsetti, Settanni, Chaves-López, et al., 2007). Starting from flour mixed with water, the selection of the non-Lactobacillus species occurs under the first repeated propagations (refreshments) of the mass, but when lactobacilli dominate their levels generally decrease at about $2-3$ orders of magnitude (Corsetti, Settanni, Valmorri, et al., 2007). However, some 


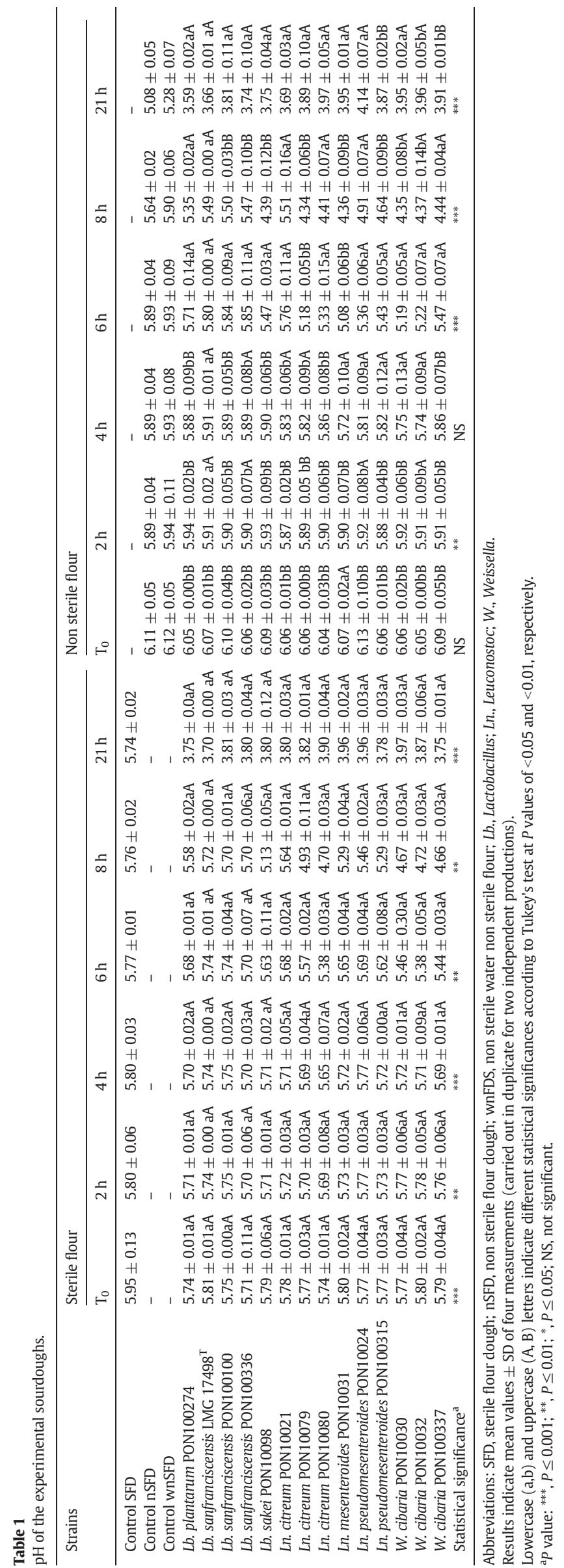

leuconostocs, pediococci and weissellas were found to coexist at the same levels of lactobacilli in sourdoughs (Robert, Gabriel, \& FontagnéFaucher, 2009).

In a previous work of ours (Alfonzo et al., 2013), 50 strains of LAB isolated from different flour samples employed to produce sourdoughs in bakeries located in the Sicily region (southern Italy) were characterised in vitro for their technological characteristics with 11 strains belonging to Leuconostoc citreum, Leuconostoc pseudomesenteroides, Leuconostoc mesenteroides, Lactobacillus plantarum, Lactobacillus sakei and Weissella cibaria showing potential in sourdough fermentation. The present work was aimed to evaluate the technological performances (acidification kinetics, acid production, loaf height, colour formation, softness of bread and volatile compound generation) of these strains under controlled conditions. To this end, the fermentation tests were carried out at first in $\gamma$-ray treated wheat flour, in order to investigate the behaviour of each strain without any microbial competition, and then in untreated flour to monitor their role in the presence of the indigenous microbiota of flour.

\section{Materials and methods}

\subsection{Strains and growth conditions}

In this study, eleven LAB strains (Lactobacillus plantarum PON100274, Lactobacillus sakei PON10098, Leuconostoc citreum PON10021, PON10079 and PON10080, Leuconostoc mesenteroides PON10031, Leuconostoc pseudomesenteroides PON10024 and PON100315 and Weissella cibaria PON10030, PON10032 and PON100337), which were isolated from wheat samples (Triticum durum and T. aestivum) and selected as technologically relevant in sourdough fermentation (Alfonzo et al., 2013), were used. They were taken from the culture collection of the Agricultural Microbiology laboratory at the Department of Agricultural and Forest Science - University of Palermo (Palermo, Italy). In addition, two Lactobacillus sanfranciscensis strains (PON100100 and PON100336) belonging to the same culture collection and $L b$. sanfranciscensis LMG $17498^{\mathrm{T}}$ were used for comparison. $\mathrm{Lb}$. sanfranciscensis strains were propagated in sourdough bacteria (SDB) broth (Kline \& Sugihara, 1971), while all other LAB strains in MRS (Oxoid, Milan, Italy). All the strains were incubated overnight at $30^{\circ} \mathrm{C}$.

\subsection{Dough production}

The LAB strains were individually tested in experimental sourdoughs in order to evaluate their in situ performances. Overnight LAB cultures, grown in the corresponding optimal media, were centrifuged at $5000 \times \mathrm{g}$ for $5 \mathrm{~min}$, washed twice in Ringer's solution (Oxoid) and re-suspended in the same solution till reaching an optical density (OD) of ca. 1.00 which approximately corresponds to a concentration of $10^{9} \mathrm{CFU} \mathrm{mL}-1$. The inocula were added to a final concentration of approximately $10^{6} \mathrm{CFU} / \mathrm{g}$ in dough.

Each dough of $200 \mathrm{~g}$ was produced with a dough yield (weight of the dough/weight of the flour $\times 100$ ) of 160 adding $75 \mathrm{~mL}$ of tap $\mathrm{H}_{2} \mathrm{O}$, containing the cell suspension, to $125 \mathrm{~g}$ of commercial flour (Il Molino Chiavazza, Casalgrasso, Italy). In order to exclude the interference of the native microflora, the experimental doughs were first prepared with wheat flour sterilised by $\gamma$-ray $(25 \mathrm{kGy})$ treatment (Gammatom, Guanzate, Italy) and mixed with sterile tap $\mathrm{H}_{2} \mathrm{O}$ (sterile flour doughs, SFD) and then the same doughs were made with untreated flour and non-sterile tap $\mathrm{H}_{2} \mathrm{O}$ (non-sterile flour doughs, nSFD). Control doughs, without $\mathrm{LAB}$ inocula, were added to each dough production: sterile flour and sterile tap $\mathrm{H}_{2} \mathrm{O}$ for SFD (control SFD); non-sterile flour and non-sterile tap $\mathrm{H}_{2} \mathrm{O}$ for nSFD (control nSFD); and, in order to exclude the contribution of $\mathrm{H}_{2} \mathrm{O}$ microflora to the dough, an additional control dough was prepared with non-sterile flour and sterile tap $\mathrm{H}_{2} \mathrm{O}$ (control wnSFD). 


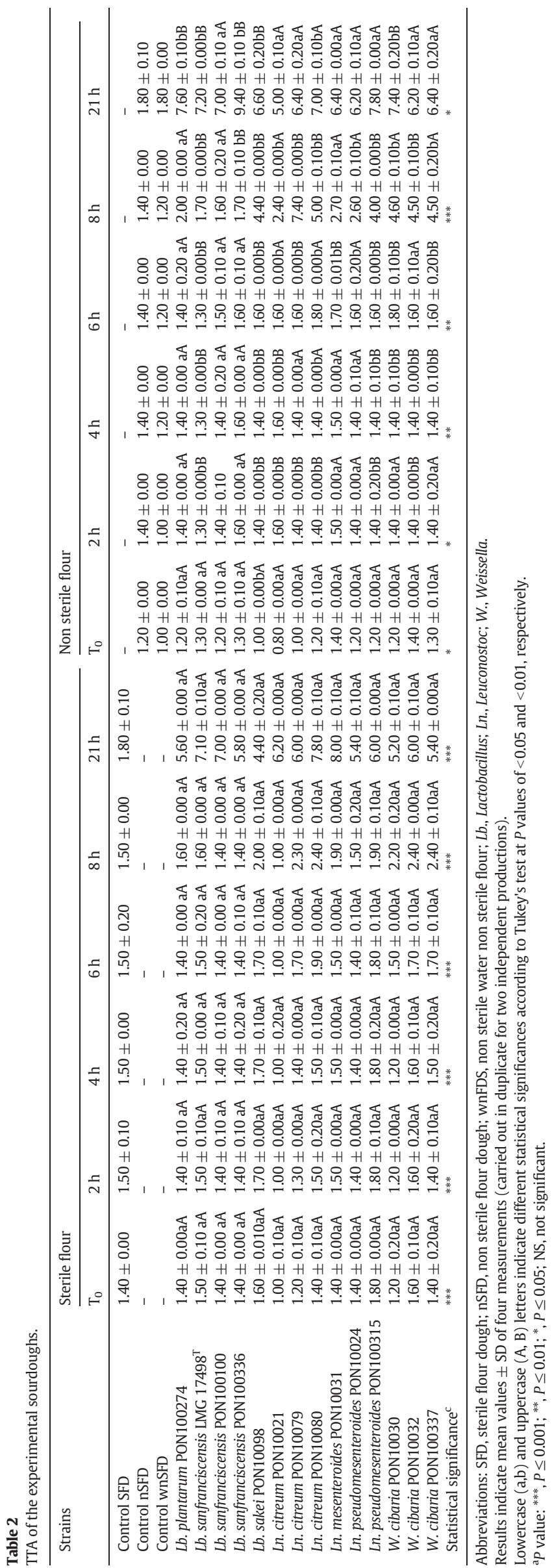

Each dough was divided in two portions: one portion of $80 \mathrm{~g}$ was placed in a stainless steel circular baking pan ( $10 \mathrm{~cm}$ diameter) covered with aluminium foil, incubated at $30{ }^{\circ} \mathrm{C}$ for $8 \mathrm{~h}$ and cooked in the industrial convection oven Modular 80012 DH (Tornati Forno S.r.l, Montelabbate, Italy), at $218{ }^{\circ} \mathrm{C}$ for $20 \mathrm{~min}$; the other portion of $120 \mathrm{~g}$ was placed in a sterile plastic beaker covered with parafilm and incubated at $30^{\circ} \mathrm{C}$ for $21 \mathrm{~h}$. Both dough productions were carried out in duplicate over two consecutive weeks.

\subsection{Sourdough analysis}

\subsection{1. $\mathrm{pH}$ and total titratable acidity}

Sourdough fermentation was followed by $\mathrm{pH}$ [determined electrometrically using the pH meter BASIC $20+$ (Crison Instrument S.A., Barcelona, Spain)] and total titratable acidity (TTA, determined by titration with $0.1 \mathrm{~N} \mathrm{NaOH}$ and expressed in terms of $\mathrm{mL}$ of $\mathrm{NaOH}$ ) tests on $5 \mathrm{~g}$ of each sample collected soon after mixing, at $2 \mathrm{~h}$ intervals for the first $8 \mathrm{~h}$ and then again after $21 \mathrm{~h}$.

\subsubsection{Microbiological analysis}

The microbial loads were determined immediately after mixing and at 8 and $21 \mathrm{~h}$ of fermentation. Ten grams of each sample was suspended in $90 \mathrm{~mL}$ of Ringer's solution, homogenised in a stomacher (BagMixer ${ }^{\circledR}$ 400, Interscience, Saint Nom, France) for 2 min at the highest speed and serially diluted. Depending on the LAB inoculated as starter culture, plate counts were performed using SDB agar or MRS agar in addition to plate count agar (PCA) (Oxoid). The microbial suspensions were plated and incubated as follows: total mesophilic count (TMC) on PCA, incubated aerobically at $30{ }^{\circ} \mathrm{C}$ for $72 \mathrm{~h}$; Lb. sanfranciscensis on SDB agar, incubated aerobically for $48 \mathrm{~h}$ at $30^{\circ} \mathrm{C}$, all other LAB on MRS agar, incubated anaerobically for $48 \mathrm{~h}$ at $30^{\circ} \mathrm{C}$. Microbiological counts were carried out in duplicate.

The presence of the microorganisms added as starter cultures was confirmed, after colony isolation from the highest dilution of sample suspensions, by microscopic inspection and randomly amplified polymorphic DNA (RAPD) analysis performed as reported by Settanni, Miceli, Francesca, and Moschetti (2012).

\subsubsection{Organic acids}

Lactic and acetic acid concentration was determined after $8 \mathrm{~h}$ of fermentation. Ten grams of each sourdough was homogenised with $90 \mathrm{~mL}$ distilled $\mathrm{H}_{2} \mathrm{O}$ by stomacher and aliquots of $10 \mathrm{~mL}$ were added with $5 \mathrm{~mL}$ of $0.1 \mathrm{mmol} / \mathrm{L} \mathrm{HClO}_{4}$ solution. The mixtures were centrifuged at $4.000 \times g$ for $15 \mathrm{~min}$ at $15^{\circ} \mathrm{C}$ and the supernatants were acidified to $\mathrm{pH} 3.0 \pm 0.1$ with $1 \mathrm{mmol} / \mathrm{L} \mathrm{HClO}_{4}$ and brought to the final volume of $25 \mathrm{~mL}$ with distilled $\mathrm{H}_{2} \mathrm{O}$. The solutions were left in ice for $30 \mathrm{~min}$ and filtered through $0.45 \mu \mathrm{m}$ cellulose filters (Millipore). HPLC analyses were conducted as reported by Alfonzo et al. (2013). PerkinElmer software specific to the HPLC instrument (TotalChrom Workstation 2008 rev. 6.3.2) was used to acquire and process data. Analyses were carried out in triplicate and the results expressed as means \pm standard deviation.

\subsubsection{Volatile organic compounds}

The volatile organic compounds (VOC) of the sourdoughs were determined after $8 \mathrm{~h}$ of fermentation. A solid phase micro extraction (SPME) isolation technique was used. Five grams of sourdough were heated to $60{ }^{\circ} \mathrm{C}$ in a vial and the headspace was collected by a DBVCarboxen-PDMS fibers (Supelco, Bellefonte, PA) for $40 \mathrm{~min}$. The SPME fibre was inserted directly into a Finnegan Trace MS for GC/MS (Agilent 6890 Series GC system, Agilent 5973 Net Work Mass Selective Detector, Milan, Italy) equipped with a DB-WAX capillary column (Agilent Technologies, $30 \mathrm{~m}, 0.250 \mathrm{~mm}$ i.d., film thickness $0.25 \mu \mathrm{m}$, part $\mathrm{n}^{\circ} 122$ 7032 ) and the analyses were conducted as reported by Alfonzo et al. (2013). 
Table 3

Microbial loads ( $\log \mathrm{CFU} / \mathrm{g}$ ) of the experimental sourdoughs.

\begin{tabular}{|c|c|c|c|c|c|c|c|c|c|c|c|c|}
\hline \multirow[t]{3}{*}{ Strains } & \multicolumn{6}{|l|}{ Sterile flour } & \multicolumn{6}{|l|}{ Non sterile flour } \\
\hline & \multicolumn{2}{|l|}{$\mathrm{T}_{0}$} & \multicolumn{2}{|l|}{$8 \mathrm{~h}$} & \multicolumn{2}{|l|}{$21 \mathrm{~h}$} & \multicolumn{2}{|l|}{$\mathrm{T}_{0}$} & \multicolumn{2}{|l|}{$8 \mathrm{~h}$} & \multicolumn{2}{|l|}{$21 \mathrm{~h}$} \\
\hline & PCA & $\begin{array}{l}\text { Specific } \\
\text { medium }\end{array}$ & PCA & $\begin{array}{l}\text { Specific } \\
\text { medium }\end{array}$ & PCA & $\begin{array}{l}\text { Specific } \\
\text { medium }\end{array}$ & PCA & $\begin{array}{l}\text { Specific } \\
\text { medium }\end{array}$ & PCA & $\begin{array}{l}\text { Specific } \\
\text { medium }\end{array}$ & PCA & $\begin{array}{l}\text { Specific } \\
\text { medium }\end{array}$ \\
\hline Control SFD & $<2$ & $\begin{array}{l}\mathrm{MRS}<1 \\
\mathrm{SDB}<2\end{array}$ & $<2$ & $\begin{array}{l}\mathrm{MRS}<1 \\
\mathrm{SDB}<2\end{array}$ & $<2$ & $\begin{array}{l}\mathrm{MRS}<1 \\
\mathrm{SDB}<2\end{array}$ & & & & & & \\
\hline Control nSFD & & & & & & & $3.00 \pm 0.02$ & $\begin{array}{l}\mathrm{MRS}<1 \\
\mathrm{SDB}<2\end{array}$ & $5.81 \pm 0.03$ & $\begin{array}{l}\text { MRS4.71 } \pm 0.03 \\
\text { SDB5.35 } \pm 0.04\end{array}$ & $6.21 \pm 0.06$ & $\begin{array}{l}\text { MRS5.77 } \pm 0.02 \\
\text { SDB6.71 } \pm 0.03\end{array}$ \\
\hline Control wnSFD & & & & & & & $3.36 \pm 0.04$ & $\begin{array}{l}\mathrm{MRS}<1 \\
\mathrm{SDB}<2\end{array}$ & $6.12 \pm 0.01$ & $\begin{array}{l}\text { MRS4.47 } \pm 0.02 \\
\text { SDB5.26 } \pm 0.06\end{array}$ & $6.13 \pm 0.05$ & $\begin{array}{l}\text { MRS5.77 } \pm 0.01 \\
\text { SDB6.78 } \pm 0.03\end{array}$ \\
\hline Lb. plantarum PON100274 & $6.05 \pm 0.05 \mathrm{aA}$ & $6.14 \pm 0.02 \mathrm{aA}$ & $7.15 \pm 0.01 \mathrm{aA}$ & $7.26 \pm 0.08 \mathrm{aA}$ & $8.20 \pm 0.05 \mathrm{aA}$ & $8.44 \pm 0.01 \mathrm{aA}$ & $6.62 \pm 0.04 \mathrm{aA}$ & $6.95 \pm 0.02 \mathrm{bB}$ & $7.81 \pm 0.02 \mathrm{bB}$ & $8.50 \pm 0.03 \mathrm{bB}$ & $8.56 \pm 0.03 \mathrm{aA}$ & $8.74 \pm 0.05 b B$ \\
\hline Lb. sanfranciscensis LMG $17498^{\mathrm{T}}$ & $6.11 \pm 0.01 \mathrm{aA}$ & $6.19 \pm 0.02 \mathrm{aA}$ & $7.02 \pm 0.02 \mathrm{aA}$ & $7.30 \pm 0.03 \mathrm{aA}$ & $7.36 \pm 0.01 \mathrm{aA}$ & $7.93 \pm 0.03 \mathrm{aA}$ & $6.27 \pm 0.02 \mathrm{aA}$ & $6.46 \pm 0.03 \mathrm{aA}$ & $7.29 \pm 0.01 \mathrm{aA}$ & $7.30 \pm 0.01 \mathrm{aA}$ & $7.69 \pm 0.02 \mathrm{aA}$ & $7.96 \pm 0.03 \mathrm{aA}$ \\
\hline Lb. sanfranciscensis PON100100 & $6.44 \pm 0.01 \mathrm{aA}$ & $6.60 \pm 0.0 \mathrm{aA}$ & $6.96 \pm 0.09 \mathrm{aA}$ & $7.10 \pm 0.01 \mathrm{aA}$ & $7.46 \pm 0.03 \mathrm{aA}$ & $8.20 \pm 0.04 \mathrm{aA}$ & $6.25 \pm 0.09 \mathrm{aA}$ & $6.50 \pm 0.05 \mathrm{aA}$ & $6.83 \pm 0.07 \mathrm{aA}$ & $7.34 \pm 0.08 \mathrm{aA}$ & $8.09 \pm 0.07 \mathrm{bB}$ & $8.29 \pm 0.02 \mathrm{aA}$ \\
\hline Lb. sanfranciscensis PON100336 & $6.23 \pm 0.02 \mathrm{aA}$ & $6.48 \pm 0.03 \mathrm{aA}$ & $6.80 \pm 0.01 \mathrm{aA}$ & $7.20 \pm 0.02 \mathrm{aA}$ & $7.27 \pm 0.04 \mathrm{aA}$ & $7.78 \pm 0.03 \mathrm{aA}$ & $6.39 \pm 0.09 \mathrm{aA}$ & $6.52 \pm 0.01 \mathrm{aA}$ & $7.33 \pm 0.03 \mathrm{bB}$ & $7.70 \pm 0.02 \mathrm{aA}$ & $8.43 \pm 0.03 \mathrm{bB}$ & $8.95 \pm 0.07 \mathrm{bB}$ \\
\hline Lb. sakei PON10098 & $6.52 \pm 0.05 \mathrm{aA}$ & $6.74 \pm 0.01 \mathrm{aA}$ & $8.64 \pm 0.07 \mathrm{aA}$ & $8.82 \pm 0.09 \mathrm{aA}$ & $8.78 \pm 0.01 \mathrm{aA}$ & $9.11 \pm 0.07 \mathrm{aA}$ & $6.16 \pm 0.05 \mathrm{aA}$ & $6.90 \pm 0.06 \mathrm{aA}$ & $8.53 \pm 0.02 \mathrm{bB}$ & $9.04 \pm 0.02 \mathrm{bB}$ & $8.58 \pm 0.03 \mathrm{bB}$ & $9.21 \pm 0.01 \mathrm{bB}$ \\
\hline Ln. citreum PON10021 & $6.59 \pm 0.01 \mathrm{aA}$ & $6.60 \pm 0.02 \mathrm{aA}$ & $7.67 \pm 0.01 \mathrm{aA}$ & $7.78 \pm 0.02 \mathrm{aA}$ & $8.54 \pm 0.03 \mathrm{aA}$ & $8.60 \pm 0.02 \mathrm{aA}$ & $6.03 \pm 0.03 \mathrm{aA}$ & $6.11 \pm 0.09 \mathrm{aA}$ & $7.44 \pm 0.06 \mathrm{bB}$ & $8.32 \pm 0.04 \mathrm{bB}$ & $8.94 \pm 0.06 \mathrm{bB}$ & $9.33 \pm 0.05 \mathrm{aA}$ \\
\hline Ln. citreum PON10079 & $6.05 \pm 0.09 \mathrm{aA}$ & $6.18 \pm 0.06 \mathrm{a} A$ & $8.07 \pm 0.09 \mathrm{aA}$ & $8.63 \pm 0.03 \mathrm{aA}$ & $8.56 \pm 0.10 \mathrm{aA}$ & $8.96 \pm 0.02 \mathrm{aA}$ & $6.03 \pm 0.07 \mathrm{aA}$ & $6.85 \pm 0.02 \mathrm{bB}$ & $8.06 \pm 0.05 \mathrm{aA}$ & $8.75 \pm 0.01 \mathrm{bB}$ & $8.27 \pm 0.02 \mathrm{bB}$ & $8.97 \pm 0.03 \mathrm{aA}$ \\
\hline Ln. citreum PON10080 & $6.17 \pm 0.02 \mathrm{aA}$ & $6.30 \pm 0.02 \mathrm{aA}$ & $8.50 \pm 0.01 \mathrm{aA}$ & $9.10 \pm 0.10 \mathrm{aA}$ & $8.58 \pm 0.07 \mathrm{aA}$ & $9.21 \pm 0.05 \mathrm{aA}$ & $6.59 \pm 0.02 \mathrm{aA}$ & $6.80 \pm 0.04 \mathrm{aA}$ & $8.48 \pm 0.04 \mathrm{aA}$ & $9.23 \pm 0.05 \mathrm{bB}$ & $9.34 \pm 0.08 \mathrm{bB}$ & $9.38 \pm 0.03 \mathrm{bB}$ \\
\hline Ln. mesenteroides PON10031 & $6.47 \pm 0.01 \mathrm{aA}$ & $6.60 \pm 0.00 \mathrm{aA}$ & $8.32 \pm 0.01 \mathrm{aA}$ & $8.92 \pm 0.04 \mathrm{aA}$ & $8.95 \pm 0.04 \mathrm{aA}$ & $9.24 \pm 0.01 \mathrm{aA}$ & $6.33 \pm 0.01 \mathrm{aA}$ & $6.58 \pm 0.03 \mathrm{aA}$ & $8.56 \pm 0.01 \mathrm{bA}$ & $8.90 \pm 0.07 \mathrm{aA}$ & $8.83 \pm 0.11 \mathrm{aA}$ & $8.93 \pm 0.01 \mathrm{bB}$ \\
\hline Ln. pseudomesenteroides PON10024 & $6.17 \pm 0.06 \mathrm{aA}$ & $6.50 \pm 0.03 \mathrm{aA}$ & $7.72 \pm 0.09 \mathrm{aA}$ & $7.84 \pm 0.02 \mathrm{aA}$ & $7.99 \pm 0.02 \mathrm{aA}$ & $8.11 \pm 0.03 \mathrm{aA}$ & $6.28 \pm 0.02 \mathrm{aA}$ & $6.69 \pm 0.03 \mathrm{bB}$ & $8.20 \pm 0.02 \mathrm{aA}$ & $8.83 \pm 0.03 \mathrm{bB}$ & $8.34 \pm 0.05 \mathrm{aA}$ & $9.03 \pm 0.06 \mathrm{bB}$ \\
\hline Ln. pseudomesenteroides PON100315 & $6.11 \pm 0.07 \mathrm{aA}$ & $6.24 \pm 0.05 \mathrm{aA}$ & $8.67 \pm 0.07 \mathrm{aA}$ & $8.77 \pm 0.04 \mathrm{aA}$ & $8.76 \pm 0.04 \mathrm{aA}$ & $8.96 \pm 0.05 \mathrm{aA}$ & $6.55 \pm 0.02 \mathrm{aA}$ & $6.99 \pm 0.01 \mathrm{bA}$ & $8.36 \pm 0.05 \mathrm{bB}$ & $8.79 \pm 0.03 \mathrm{aA}$ & $8.45 \pm 0.01 \mathrm{aA}$ & $8.93 \pm 0.09 \mathrm{aA}$ \\
\hline W. cibaria PON10030 & $6.63 \pm 0.06 \mathrm{aA}$ & $6.70 \pm 0.02 \mathrm{aA}$ & $7.92 \pm 0.06 \mathrm{aA}$ & $8.62 \pm 0.01 \mathrm{aA}$ & $8.57 \pm 0.02 \mathrm{aA}$ & $9.12 \pm 0.11 \mathrm{aA}$ & $6.20 \pm 0.05 \mathrm{bA}$ & $6.60 \pm 0.05 \mathrm{bB}$ & $8.77 \pm 0.02 \mathrm{bB}$ & $8.81 \pm 0.01 \mathrm{bB}$ & $8.90 \pm 0.03 \mathrm{aA}$ & $8.82 \pm 0.04 \mathrm{bB}$ \\
\hline W. cibaria PON10032 & $6.22 \pm 0.03 \mathrm{aA}$ & $6.85 \pm 0.07 \mathrm{aA}$ & $8.12 \pm 0.02 \mathrm{aA}$ & $8.18 \pm 0.05 \mathrm{aA}$ & $8.31 \pm 0.02 \mathrm{aA}$ & $8.56 \pm 0.08 \mathrm{aA}$ & $6.33 \pm 0.06 \mathrm{aA}$ & $6.76 \pm 0.09 \mathrm{bB}$ & $8.60 \pm 0.08 \mathrm{aA}$ & $9.06 \pm 0.05 \mathrm{bB}$ & $9.50 \pm 0.04 \mathrm{bA}$ & $9.72 \pm 0.03 \mathrm{bB}$ \\
\hline W. cibaria PON100337 & $6.41 \pm 0.08 \mathrm{aA}$ & $6.70 \pm 0.07 \mathrm{aA}$ & $8.20 \pm 0.01 \mathrm{aA}$ & $8.83 \pm 0.02$ & $8.51 \pm 0.04 \mathrm{aA}$ & $9.17 \pm 0.05 \mathrm{aA}$ & $6.47 \pm 0.04 \mathrm{aA}$ & $6.80 \pm 0.02 \mathrm{aA}$ & $8.18 \pm 0.04 \mathrm{aA}$ & $8.68 \pm 0.02$ & $8.58 \pm 0.01 \mathrm{aA}$ & $9.05 \pm 0.01 \mathrm{bB}$ \\
\hline Statistical significance ${ }^{c}$ & $* * *$ & $* * *$ & $* * *$ & $* * *$ & $* * *$ & $* * *$ & *** & $* * *$ & $* * *$ & $* * *$ & $* * *$ & $* * *$ \\
\hline
\end{tabular}

Abbreviations: SFD, sterile flour dough; nSFD, non sterile flour dough; wnFDS, non sterile water non sterile flour; Lb., Lactobacillus; Ln., Leuconostoc; W., Weissella.

Results indicate mean values \pm SD of four plate counts (carried out in duplicate for two independent productions).

Lowercase (a,b) and uppercase (A, B) letters indicate different statistical significances according to Tukey's test at $P$ values of $<0.05$ and $<0.01$, respectively.

a $P$ value: ***, $P \leq 0.001 ; * *, P \leq 0.01 ; *, P \leq 0.05$; NS, not significant. 
Table 4

Organic acids produced by LAB in sourdoughs processed with non sterile flour after $8 \mathrm{~h}$ of fermentation.

\begin{tabular}{|c|c|c|c|}
\hline Strains & Lactic acid (mg/g) & Acetic acid (mg/g) & FQ \\
\hline Control SFD $\left(\mathrm{T}_{0}\right)$ & 0.00 & 0.00 & \\
\hline Control SFD $\left(T_{8}\right)$ & $1.31 \pm 0.05$ & $0.15 \pm 0.01$ & 8.73 \\
\hline Control wnSFD $\left(\mathrm{T}_{0}\right)$ & $1.11 \pm 0.03$ & $0.17 \pm 0.02$ & 6.52 \\
\hline Control wnSFD $\left(\mathrm{T}_{8}\right)$ & $1.31 \pm 0.05$ & $0.25 \pm 0.03$ & 5.24 \\
\hline Lb. plantarum PON100274 & $6.47 \pm 0.02$ & $0.67 \pm 0.07$ & 9.65 \\
\hline Lb. sanfranciscensis LMG $17498^{\mathrm{T}}$ & $1.36 \pm 0.02$ & $0.30 \pm 0.04$ & 4.53 \\
\hline Lb. sanfranciscensis PON100100 & $2.24 \pm 0$ & $0.47 \pm 0.07$ & 4.77 \\
\hline Lb. sanfranciscensis PON100336 & $4.81 \pm 0.06$ & $0.61 \pm 0.02$ & 7.88 \\
\hline Lb. sakei PON10098 & $3.96 \pm 0.09$ & $0.25 \pm 0.01$ & 15.84 \\
\hline Ln. citreum PON10021 & $2.28 \pm 0.10$ & $0.25 \pm 0.03$ & 9.12 \\
\hline Ln. citreum PON10079 & $2.45 \pm 0.04$ & $0.68 \pm 0.02$ & 3.60 \\
\hline Ln. citreum PON10080 & $3.47 \pm 0.07$ & $0.87 \pm 0.13$ & 3.99 \\
\hline Ln. mesenteroides PON10031 & $2.81 \pm 0.03$ & $0.70 \pm 0.06$ & 4.01 \\
\hline Ln. pseudomesenteroides PON10024 & $4.17 \pm 0.12$ & $0.97 \pm 0.09$ & 4.30 \\
\hline Ln. pseudomesenteroides PON100315 & $3.99 \pm 0.11$ & $1.08 \pm 0.06$ & 3.69 \\
\hline W. cibaria PON10030 & $3.31 \pm 0.08$ & $0.84 \pm 0.05$ & 3.94 \\
\hline W. cibaria PON10032 & $1.42 \pm 0.02$ & $0.46 \pm 0.04$ & 3.09 \\
\hline W. cibaria PON100337 & $2.45 \pm 0.09$ & $0.40 \pm 0.02$ & 6.12 \\
\hline Statistical significance ${ }^{a}$ & $* * *$ & $* * *$ & n.d. \\
\hline
\end{tabular}

Abbreviations: SFD, sterile flour dough; nSFD, non sterile flour dough; wnFDS, non sterile water non sterile flour; Lb., Lactobacillus; Ln., Leuconostoc; W., Weissella; n.d., not determined. Results indicate mean values \pm SD of four measurements (carried out in duplicate for two independent productions).

$P$ value: ***, $P \leq 0.01$.

n.d., not determined.

All compounds in the range $\mathrm{m} / \mathrm{z} 33-495$ atomic mass unit (amu) were detected by the scan mode. The identification of the individual peaks was obtained by direct comparison of their retention indices to those of authentic samples, as well as by comparing their mass spectra with the NIST/EPA/NIH Mass Spectral Library database (Version 2.0d, build 2005). The contents of the volatile compounds were expressed as relative peak areas (peak area of each compound/total area) $\times 100$.

All solvents and reagents were purchased from WWR International (Milan, Italy). Chemical and physical determinations were performed in triplicate and the results expressed as means \pm standard deviation.

\subsection{Bread analysis}

The bread quality attributes were evaluated after cooling at ambient temperature. After weighing, each bread was cut transversely in two halves and the height of the central slice was measured (Schober, Messerschmidt, Bean, Park, \& Arendt, 2005).

Colour was measured on four points of the crust and three points of the crumb of the central slices by means of a colorimeter (Chroma Meter CR-400C, Minolta, Osaka, Japan). The Hunter's scale parameters were determined: $\mathrm{L}^{*}, \mathrm{a}^{*}$ and $\mathrm{b}^{*}$.

The hardness of crumb was determined by measuring its resistance in four points to the plunger $(6 \mathrm{~mm}$ diameter stainless steel cylinder probe) of a digital penetrometer ( $\mathrm{Tr}$ snc, Italy).

The two central slices of each loaf were scanned (Epson Perfection 4180 Photo, Seiko Epson Corp., Japan) with 350 dpi of resolution and the images were saved in TIFF format. The images were analysed with the ImageJ software (National Institutes Health, Bethesda, Md, USA). Each image was cropped to a square of $207 \times 207$ pixels (representing $15 \times 15 \mathrm{~mm}$ of the slice area) and converted to grey-level image ( $8 \mathrm{bit}$ ). A binary image was obtained applying the Otsu's threshold algorithm, in order to calculate void fraction (the fraction of the total area corresponding to the bread pores), cell density (number of cells $/ \mathrm{cm}^{2}$ ) and mean cell area in $\mathrm{mm}^{2}$.

\subsection{Statistical analyses}

Data of acidification, TTA, microbial load and organic acid concentration of sourdoughs and height, colour, hardness, void fraction, cell density and mean cell area of the resulting breads were statistically analysed using the ANOVA procedure with the software SAS 2004, version 9.1.2 (Statistical Analysis System Institute Inc., Cary, NC, USA). Differences between means were determined by Tukey's multiplerange test.

\section{Results and discussion}

\section{1. $\mathrm{pH}$, total titratable acidity and microbiological analysis of sourdoughs}

The $\mathrm{pH}$ values registered for the experimental sourdoughs produced with sterile and non-sterile flour are reported in Table 1. Although other studies carried out to evaluate the ability of different LAB strains to act as starter cultures for sourdough products (Choi, Kim, Hwang, Kim, \& Yoon, 2012; Plessas et al., 2007, 2008) tested the performances of the bacteria in untreated flour, since our study was focused on the selection of LAB, we started the screening of the strains with a sterile flour, in order to follow each culture without interference from the native microbiota within the flour.

The trials carried out with sterile flour and LAB showed an initial pH value significantly lower than the corresponding control trial (control SFD). The last dough showed only a slight $\mathrm{pH}$ decrease (from 5.95 to 5.74) during the $21 \mathrm{~h}$ of fermentation. The dough inoculated with LAB behaved similarly during the first $4 \mathrm{~h}$ of observation, while a different $\mathrm{pH}$ was registered from the $6 \mathrm{~h}$ onward: all $W$. cibaria strains and $L n$. citreum PON10080 determined a lower pH than the other trials, both at 6 and $8 \mathrm{~h}$, and their values were not statistically different. After $21 \mathrm{~h}$ of incubation, all $13 \mathrm{LAB}$ dropped the $\mathrm{pH}$ of the sourdoughs below 4.0. Regarding the doughs produced with non-sterile flours, their $\mathrm{pHs}$ were all higher than 6.0 at $\mathrm{T}_{0}$. Both control trials (with sterile and non-sterile $\mathrm{H}_{2} \mathrm{O}$ ) showed a pH decrease stronger than control dough made with sterile flour, but the value registered after $21 \mathrm{~h}$ was above 5.0. However, control nSFD showed a lower final $\mathrm{pH}$ than control wnSFD. The doughs started with non-sterile flour and LAB showed differences with the corresponding trials made with sterile flour: at $6 \mathrm{~h}$, in addition to W. cibaria PON10030 and PON10032, Ln. citreum PON10079 and Ln. mesenteroides PON10031 displayed a fast decrease in $\mathrm{pH}$ value; at $8 \mathrm{~h}$, the results of these four strains were superimposable with those of $W$. cibaria PON100337 and $L b$. sakei PON10098; except Ln. pseudomesenteroides PON10024, all other LAB determined the decrease of pH below 4.0 after $21 \mathrm{~h}$ of fermentation. In general, a similar trend was observed for the LAB used as starter cultures in both conditions, with sterile and non-sterile flour. Our results are comparable with those reported by Moroni, Arendt, and Dal Bello (2001) who followed buckwheat and teff sourdoughs spontaneously fermented with lactobacilli, leuconostocs and weissellas.

TTA data (Table 2) confirmed the observations made with the $\mathrm{pH}$ results. After $8 \mathrm{~h}$ of fermentation, all weissellas and Ln. citreum PON10079 and PON10080 were the most acidifying strains in the presence of sterile and non-sterile flour. $\mathrm{Ln}$. citreum strains applied in this work showed kinetics of acidification more rapid than that displayed by Ln. citreum HO12, of kimchii origin, tested in sourdough in conditions similar to those of our study at the same initial concentration of $10^{6} \mathrm{CFU} / \mathrm{g}$ (Choi et al., 2012).

The bacterial inocula were followed during fermentation by plate counts (Table 3 ). All bacteria in both conditions increased their cell concentrations after $8 \mathrm{~h}$. The lowest increase was registered for both $L b$. sanfranciscensis strains evaluated, while the highest values were shown by Ln. citreum PON10080. After $21 \mathrm{~h}$ of fermentation, LAB concentrations increased by at least 1.5 orders of magnitude than $T_{0}$. No major differences were found among the concentrations in the corresponding trials carried out with sterile and non-sterile flours. Control SFD was characterised by undetectable levels of total microorganisms and LAB during the whole period of fermentation; on the contrary, control nSFD and control wnSFD, although displaying undetectable levels of LAB at $\mathrm{T}_{0}$, showed levels of $10^{4}$ and $10^{5} \mathrm{CFU} / \mathrm{g}$ on 
Table 5

Analysis of volatile organic compounds emitted from sourdoughs made from untreated wheat flour inoculated with LAB and fermented for $8 \mathrm{~h}$.

\begin{tabular}{|c|c|c|c|c|c|c|c|c|c|c|c|c|c|c|c|c|c|c|}
\hline \multirow[t]{2}{*}{ Chemical compounds ${ }^{\mathrm{a}}$} & \multicolumn{18}{|c|}{ Sourdoughs ${ }^{\mathrm{b}}$} \\
\hline & $\operatorname{SFD}\left(T_{0}\right)$ & $\operatorname{SFD}\left(T_{8}\right)$ & $\operatorname{wnSFD}\left(T_{0}\right)$ & wnSFD $\left(T_{8}\right)$ & 10030 & 10032 & 10079 & 10080 & 100337 & 100336 & 100100 & 10098 & 10031 & 100274 & 100315 & 10021 & 10024 & 17498 \\
\hline Ethanol & 0.00 & 0.00 & 0.00 & 0.00 & 87.95 & 178.75 & 93.55 & 88.51 & 93.72 & 43.40 & 37.68 & 0.00 & 194.62 & 0.00 & 141.68 & 0.00 & 122.39 & 0.00 \\
\hline Hexanal & 1.05 & 1.74 & 0.36 & 1.00 & 0.35 & 1.01 & 0.43 & 0.31 & 0.49 & 1.62 & 0.46 & 0.98 & 1.07 & 0.74 & 0.25 & 0.43 & 0.19 & 0.62 \\
\hline 3-Methyl-1-butanolacetate & 0.00 & 0.00 & 0.00 & 0.00 & 0.00 & 0.18 & 0.06 & 0.33 & 1.24 & 0.00 & 0.00 & 0.00 & 0.00 & 0.00 & 0.00 & 0.00 & 0.12 & 0.00 \\
\hline 3-Methyl-1-butanol & 3.43 & 2.42 & 1.65 & 1.82 & 0.90 & 2.65 & 3.73 & 10.76 & 33.86 & 12.33 & 10.14 & 0.61 & 2.41 & 1.96 & 0.61 & 1.93 & 15.88 & 0.27 \\
\hline 1-Pentanol & 0.79 & 0.76 & 0.82 & 0.30 & 0.53 & 1.11 & 1.12 & 1.12 & 0.91 & 1.63 & 1.09 & 1.34 & 1.04 & 0.65 & 0.78 & 0.73 & 0.98 & 0.33 \\
\hline 2-Butanone-3-Hydroxy & 0.00 & 0.00 & 0.00 & 0.00 & 0.00 & 0.00 & 0.00 & 0.00 & 0.00 & 0.00 & 0.00 & 0.00 & 0.00 & 0.95 & 0.00 & 0.68 & 0.00 & 0.00 \\
\hline Tridecane & 0.43 & 0.23 & 0.89 & 0.20 & 0.00 & 0.41 & 0.00 & 0.27 & 0.00 & 0.45 & 0.18 & 0.10 & 0.00 & 0.07 & 0.82 & 0.00 & 0.00 & 0.00 \\
\hline 2-Heptenal & 0.48 & 0.24 & 0.22 & 0.29 & 0.41 & 0.18 & 0.52 & 0.28 & 0.36 & 0.45 & 0.38 & 0.41 & 0.44 & 0.14 & 0.25 & 0.49 & 0.26 & 0.22 \\
\hline Ethyl lactate & 0.00 & 0.00 & 0.00 & 0.00 & 0.62 & 0.00 & 0.00 & 0.00 & 0.00 & 0.00 & 0.00 & 0.00 & 1.22 & 0.00 & 3.21 & 0.00 & 0.88 & 0.00 \\
\hline 1-Hexanol & 5.52 & 4.11 & 5.46 & 4.05 & 9.27 & 15.01 & 11.38 & 10.93 & 11.99 & 14.13 & 9.26 & 5.99 & 15.18 & 7.25 & 6.62 & 4.91 & 8.29 & 2.34 \\
\hline Nonanal & 1.20 & 0.37 & 0.14 & 0.24 & 0.00 & 0.00 & 0.00 & 0.00 & 0.00 & 0.00 & 0.00 & 0.00 & 0.19 & 0.00 & 0.00 & 0.10 & 0.00 & 0.47 \\
\hline 2-Octenal & 0.72 & 0.44 & 0.09 & 0.28 & 0.90 & 0.89 & 0.37 & 0.34 & 0.34 & 0.45 & 0.36 & 0.64 & 0.80 & 0.60 & 0.33 & 0.00 & 0.52 & 0.35 \\
\hline 2-Pentanol & 0.04 & 0.06 & 0.07 & 0.09 & 0.06 & 0.19 & 0.10 & 0.09 & 0.14 & 0.08 & 0.08 & 0.07 & 0.10 & 0.09 & 0.07 & 0.08 & 0.11 & 0.03 \\
\hline Ethyl octanoate & 0.18 & 0.00 & 0.03 & 0.05 & 0.00 & 0.00 & 0.00 & 0.00 & 0.00 & 0.00 & 0.00 & 0.00 & 0.00 & 0.00 & 0.04 & 0.02 & 0.19 & 0.00 \\
\hline Acetic acid & 0.24 & 0.30 & 0.60 & 0.25 & 7.74 & 5.11 & 14.66 & 11.56 & 10.57 & 1.93 & 1.51 & 1.21 & 17.91 & 0.84 & 17.16 & 0.95 & 7.40 & 0.05 \\
\hline 1-Octen-3-ol & 1.96 & 1.42 & 1.17 & 1.40 & 1.30 & 1.87 & 1.61 & 1.47 & 2.04 & 2.41 & 1.73 & 1.43 & 2.26 & 1.39 & 1.81 & 1.45 & 1.73 & 0.82 \\
\hline 1-Heptanol & 0.75 & 0.50 & 0.48 & 0.49 & 1.07 & 1.51 & 1.18 & 1.03 & 1.32 & 1.02 & 0.99 & 0.53 & 1.10 & 0.77 & 1.03 & 0.58 & 0.95 & 0.23 \\
\hline 6-Methyl-5-hepten-2-ol & 0.19 & 0.09 & 0.16 & 0.13 & 0.20 & 0.23 & 0.17 & 0.17 & 0.20 & 0.24 & 0.14 & 0.12 & 0.16 & 0.12 & 0.19 & 0.10 & 0.14 & 0.03 \\
\hline 2-Ethylhexanol & 0.97 & 0.46 & 0.87 & 0.61 & 0.45 & 0.87 & 0.52 & 0.46 & 0.38 & 0.66 & 0.67 & 0.00 & 0.58 & 0.57 & 0.53 & 0.61 & 0.49 & 0.21 \\
\hline Benzaldehyde & 2.63 & 2.18 & 1.71 & 2.49 & 3.25 & 0.91 & 3.29 & 5.08 & 1.92 & 1.47 & 1.93 & 1.26 & 2.14 & 3.50 & 3.04 & 2.11 & 2.15 & 1.84 \\
\hline 2-Hepten-1-ol & 0.00 & 0.06 & 0.00 & 0.00 & 0.47 & 0.73 & 1.02 & 0.62 & 0.92 & 0.44 & 0.27 & 0.90 & 0.76 & 0.00 & 0.71 & 0.00 & 0.56 & 0.03 \\
\hline 2-Nonenal & 0.98 & 1.27 & 0.39 & 1.49 & 1.42 & 0.34 & 0.68 & 0.74 & 0.54 & 0.57 & 0.63 & 0.00 & 0.42 & 1.20 & 0.91 & 0.00 & 1.06 & 0.79 \\
\hline Propanoic acid & 0.11 & 3.05 & 0.10 & 0.85 & 0.93 & 0.00 & 0.00 & 0.00 & 0.00 & 0.00 & 0.00 & 0.00 & 0.13 & 0.00 & 0.36 & 0.86 & 0.71 & 0.00 \\
\hline Ethyl-2-Hydroxyhexanoate & 0.00 & 0.00 & 0.00 & 0.00 & 0.00 & 0.04 & 0.08 & 0.04 & 0.17 & 0.00 & 0.04 & 0.00 & 0.00 & 0.00 & 0.10 & 0.00 & 0.03 & 0.00 \\
\hline 3,5-Octadien-2-one & 0.14 & 0.11 & 0.05 & 0.14 & 0.11 & 0.15 & 0.04 & 0.04 & 0.10 & 0.17 & 0.10 & 0.06 & 0.00 & 0.08 & 0.09 & 0.18 & 0.04 & 0.12 \\
\hline 1-Octanol & 0.92 & 0.58 & 0.43 & 0.59 & 0.69 & 1.07 & 0.70 & 0.42 & 0.65 & 0.70 & 0.71 & 0.47 & 0.79 & 0.59 & 0.86 & 0.60 & 0.59 & 0.38 \\
\hline g-Butyrolactone & 0.10 & 0.10 & 0.10 & 0.05 & 0.08 & 0.14 & 0.12 & 0.18 & 0.22 & 0.18 & 0.19 & 0.17 & 0.13 & 0.16 & 0.08 & 0.04 & 0.07 & 0.03 \\
\hline
\end{tabular}




\begin{tabular}{|c|c|c|c|c|c|c|c|c|c|c|c|c|c|c|c|c|c|c|}
\hline 2-Octen-1-ol & 0.00 & 1.86 & 0.00 & 0.31 & 0.57 & 0.60 & 0.00 & 0.73 & 0.80 & 0.28 & 0.22 & 0.00 & 0.35 & 0.00 & 0.69 & 0.26 & 0.67 & 0.00 \\
\hline 3-Nonen-1-ol & 0.11 & 1.61 & 0.00 & 0.70 & 0.68 & 0.90 & 1.67 & 0.59 & 0.78 & 0.27 & 0.20 & 0.64 & 1.53 & 0.00 & 0.76 & 0.60 & 0.64 & 0.18 \\
\hline Dodecanal & 0.00 & 0.00 & 0.00 & 0.00 & 0.00 & 0.13 & 0.25 & 0.48 & 2.43 & 0.84 & 0.95 & 0.34 & 0.00 & 0.00 & 0.00 & 0.00 & 0.00 & 0.00 \\
\hline 2-Methylbenzaldehyde & 0.00 & 0.00 & 0.00 & 0.00 & 0.00 & 0.22 & 0.00 & 0.00 & 0.49 & 0.19 & 0.21 & 0.17 & 0.07 & 0.00 & 0.00 & 0.00 & 0.00 & 0.00 \\
\hline 2-Nonen-1-ol & 0.00 & 0.00 & 0.00 & 0.00 & 0.00 & 0.28 & 0.00 & 0.00 & 0.61 & 0.31 & 0.30 & 0.00 & 0.47 & 0.00 & 0.00 & 0.00 & 0.00 & 0.00 \\
\hline Phenylmethylacetate & 0.39 & 0.00 & 0.00 & 0.00 & 1.01 & 0.00 & 2.17 & 2.86 & 1.25 & 0.00 & 0.00 & 0.00 & 0.00 & 0.24 & 0.78 & 0.00 & 0.82 & 0.00 \\
\hline Pentanoic acid & 0.00 & 0.00 & 0.00 & 0.00 & 0.16 & 0.27 & 0.21 & 0.09 & 0.13 & 0.12 & 0.07 & 0.20 & 0.31 & 0.12 & 0.21 & 0.05 & 0.14 & 0.00 \\
\hline 2,4-Decadienal & 0.19 & 0.13 & 0.04 & 0.00 & 0.42 & 0.69 & 0.21 & 0.07 & 0.13 & 0.16 & 0.10 & 0.12 & 0.63 & 0.24 & 0.37 & 0.00 & 0.08 & 0.12 \\
\hline Methylnaphthalene & 0.00 & 0.03 & 0.00 & 0.00 & 0.07 & 0.06 & 3.99 & 3.51 & 0.08 & 0.05 & 0.04 & 7.05 & 0.00 & 0.00 & 0.00 & 0.00 & 0.00 & 0.00 \\
\hline Hexanoic acid & 0.00 & 0.00 & 0.00 & 0.00 & 5.18 & 5.40 & 2.78 & 2.29 & 2.46 & 6.53 & 4.71 & 0.00 & 7.69 & 2.89 & 5.45 & 9.44 & 4.31 & 0.00 \\
\hline Benzyl alcohol & 22.38 & 16.02 & 11.24 & 9.71 & 57.10 & 15.74 & 64.37 & 121.52 & 24.69 & 8.19 & 25.82 & 10.14 & 10.33 & 67.33 & 76.01 & 19.74 & 48.47 & 14.28 \\
\hline Phenylethyl Alcohol & 0.58 & 0.23 & 0.10 & 0.18 & 0.24 & 0.39 & 0.33 & 0.30 & 0.35 & 0.32 & 0.35 & 0.29 & 0.20 & 0.41 & 0.27 & 0.19 & 0.17 & 0.33 \\
\hline Tridecanal & 0.00 & 0.00 & 0.00 & 0.00 & 0.00 & 0.46 & 0.00 & 0.22 & 0.94 & 0.00 & 0.21 & 0.19 & 0.00 & 0.00 & 0.00 & 0.00 & 0.00 & 0.00 \\
\hline 1,4-Butanediol & 0.04 & 0.05 & 0.07 & 0.00 & 0.09 & 0.29 & 0.13 & 0.13 & 0.22 & 0.22 & 0.10 & 0.10 & 0.12 & 0.07 & 0.11 & 0.00 & 0.03 & 0.00 \\
\hline 2-Ethylhexanoic acid & 0.00 & 0.04 & 0.00 & 0.00 & 0.05 & 0.28 & 0.02 & 0.19 & 0.19 & 0.12 & 0.01 & 0.14 & 0.19 & 0.17 & 0.10 & 0.05 & 0.07 & 0.00 \\
\hline Phenol & 0.15 & 0.23 & 0.20 & 0.20 & 0.28 & 0.38 & 0.20 & 0.23 & 0.29 & 0.24 & 0.24 & 0.22 & 0.54 & 0.27 & 0.26 & 0.19 & 0.15 & 0.09 \\
\hline g-Nonalactone & 0.31 & 0.32 & 0.36 & 0.32 & 0.36 & 0.49 & 0.29 & 0.27 & 0.33 & 0.51 & 0.33 & 0.29 & 0.38 & 0.30 & 0.45 & 0.40 & 0.35 & 0.13 \\
\hline p-Phthalaldehyde & 0.12 & 0.12 & 0.24 & 0.12 & 0.13 & 0.31 & 0.29 & 0.30 & 0.38 & 0.41 & 0.38 & 0.23 & 0.33 & 0.24 & 0.15 & 0.12 & 0.07 & 0.00 \\
\hline Isophthalaldehyde & 0.09 & 0.10 & 0.13 & 0.06 & 0.23 & 0.20 & 0.26 & 0.29 & 0.36 & 0.39 & 0.38 & 0.24 & 0.19 & 0.20 & 0.11 & 0.05 & 0.04 & 0.26 \\
\hline Pentadecanal & 0.05 & 0.00 & 0.13 & 0.00 & 0.00 & 0.11 & 0.13 & 0.14 & 1.18 & 0.28 & 0.36 & 0.08 & 0.11 & 0.00 & 0.14 & 0.09 & 0.04 & 0.00 \\
\hline Ethylhexadecanoate & 0.00 & 0.00 & 0.00 & 0.00 & 0.09 & 0.12 & 0.04 & 0.03 & 0.09 & 0.00 & 0.14 & 0.00 & 0.12 & 0.00 & 0.05 & 0.04 & 0.09 & 0.00 \\
\hline Diethylphthalate & 0.54 & 0.04 & 0.06 & 0.00 & 1.16 & 0.13 & 0.12 & 0.13 & 1.38 & 0.28 & 0.24 & 0.11 & 0.00 & 0.08 & 0.09 & 0.00 & 0.00 & 0.22 \\
\hline Benzoic acid & 0.04 & 0.10 & 0.00 & 0.42 & 0.11 & 0.00 & 0.06 & 0.09 & 0.11 & 0.04 & 0.10 & 0.14 & 0.11 & 0.10 & 0.04 & 0.04 & 0.00 & 0.00 \\
\hline Benzophenone & 0.24 & 0.10 & 0.07 & 0.18 & 0.14 & 0.11 & 0.09 & 0.14 & 0.14 & 0.11 & 0.10 & 0.18 & 0.22 & 0.10 & 0.19 & 0.08 & 0.00 & 0.05 \\
\hline
\end{tabular}

Results indicate mean values of four measurements (carried out in duplicate for two independent productions) and are expressed as relative peak areas (peak area of each compound/total area) $\times 100$.

a The chemicals are shown following their retention time.
Theate

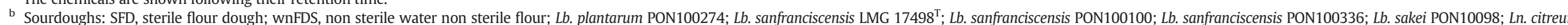
PON10021; Ln. citreum PON10079; Ln. citreum PON10080; Ln. mesenteroides PON10031; Ln. pseudomesenteroides PON10024; Ln. pseudomesenteroides PON100315; W. cibaria PON10030; W. cibaria PON10032; W. cibaria PON100337. 
MRS and SDB, respectively, at $8 \mathrm{~h}$ and $10^{5}$ and $10^{6} \mathrm{CFU} / \mathrm{g}$ on MRS and SDB, respectively, after $21 \mathrm{~h}$. Thus, the levels of $L A B$ inoculated with the selected strains were not affected by the indigenous LAB of commercial flour and reached the typical final concentrations obtained with similar approaches (Choi et al., 2012; Moroni et al., 2001). However, the conditions of sourdough propagation might influence the interactions between LAB (Corsetti, Settanni, Valmorri, et al., 2007). Minervini, Lattanzi, De Angelis, Di Cagno, and Gobbetti (2012) reported that the LAB populations differed among sourdoughs propagated at artisan bakery and laboratory levels: $L b$. plantarum, $L b$. sakei, and W. cibaria dominated in some sourdoughs back-slopped at artisan bakeries, while Ln. citreum were more persistent under laboratory conditions.

\subsection{Bacterial comparison}

The isolates collected from MRS and SDB resulting from the plate counts performed at $8 \mathrm{~h}$, at the highest dilutions of samples, were analysed at strain level by means of RAPD-PCR with primer M13. Amplified DNAs from the isolates of a given trial, together with that of the pure culture corresponding to the same trial, were loaded onto a gel in order to recognise the added bacteria through fermentation and to evaluate their contribution to the concentrations estimated. The direct comparison of the RAPD patterns (results not shown) allowed the recognition of the added cultures in both conditions of flour used. The RAPD profiles of the LAB isolated at the highest concentrations from the control doughs made with non-sterile flour (results not shown) excluded the presence of any of the 13 LAB used in the commercial flour in this study.

The use of RAPD analysis to monitor the dominance and/or the persistence of added strains is commonly applied in sourdough preparation and propagation (Ehrmann \& Vogel, 2005; Minervini et al., 2010; Settanni, Massitti, Van Sinderen, \& Corsetti, 2005) and our results highlighted the relevance of this technique to rapidly monitor the fermenting LAB that are responsible for the successful sourdough fermentation.

\subsection{Chemical analysis of sourdoughs}

Organic acid production and VOC generation were determined only for the sourdoughs obtained with the non-sterile flour, in order to evaluate the contribution of each LAB in production conditions.

The concentrations of the organic acids and the resulting FQ of the doughs are reported in Table 4. Lactic acid was in the range 1.36 $6.47 \mathrm{mg} / \mathrm{g}$ with the lowest value shown by $\mathrm{Lb}$. sanfranciscensis LMG $17498^{\mathrm{T}}$ and the highest by $L b$. plantarum PON100274. The lowest value of acetic acid was $0.25 \mathrm{mg} / \mathrm{g}$ and the highest $1.08 \mathrm{mg} / \mathrm{g}$ as registered for Lb. sakei PON10098 and Ln. pseudomesenteroides PON100315, respectively. FQ ranged between 3.09 and 15.84; the doughs started with Ln. citreum PON10079 and PON 10080, Ln. pseudomesenteroides PON100315 and $W$. cibaria PON10030 and PON10032 were characterised by a FQ comprised in the range 1.5 - 4 that is considered to affect positively the aroma profile and the structure of the final products (Spicher, 1983), but only W. cibaria PON10032 determined an FQ close to the optimal range of $2.0-2.7$ suggested by Hammes and Gänzle (1998). Lactic acid can gradually account for a more elastic gluten structure. Acetic acid, produced by heterofermentative LAB, is responsible for a shorter and harder gluten (Lorenz, 1983) and shows antirope and antimould effects (Rosenquist \& Hansen, 1998).

VOC composition resulting from the chromatographic analysis is reported in Table 5. In the headspace of sourdoughs, 51 compounds were identified: 5 acids, 16 alcohols, 13 aldehydes, 1 acetate, 1 alkane, 9 esters, 3 ketones, 2 lactones and 1 phenol. Several compounds, whose presence is associated with the metabolism of $\mathrm{LAB}$, are able to affect the final bread since may provide unique flavours that contribute to a pleasant aroma and taste (Hansen \& Hansen, 1996). Most of the compounds identified in the experimental sourdoughs are reported to be relevant for the breads processed with this technology (Hansen \& Lund, 1987; Seitz, Chung, \& Rengarajan, 1998), even though not all volatile compounds detected by instrumental analysis have a perceptible aroma (Meignen et al., 2001). The compounds that strongly affect bread flavour are mainly organic acids, alcohols, esters and carbonyls (Czerny \& Schieberle, 2002; Kirchhoff \& Schieberle, 2002).

Except in doughs started with $L b$. sakei PON 10098, Lb. plantarum PON100274, Ln. citreum PON10021 and Lb. sanfranciscensis LMG $17498^{\mathrm{T}}$, ethanol was the VOC quantitatively most present in all other doughs. However, the amounts of ethanol revealed should not particularly affect the bread quality because the ethanol produced by baker's yeast is much higher than the level produced by LAB (Choi et al., 2012). Benzyl alcohol was the second VOC in terms of concentration, followed by 1hexanol. Although ethanol was detected only after the 8-h fermentation period, benzyl alcohol and 1-hexanol were already present in the nonfermented doughs. Among the acid component of VOC, acetic acid was that produced at the highest level for the majority of doughs. The dough inoculated with $W$. cibaria PON10030 showed the highest concentrations of 2-nonenal and propanoic acid. Ln. citreum PON10079 and PON10080 determined the highest level of phenylmethylacetate. Although nonanal is reported to be present at high amounts in sourdough breads (Seitz et al., 1998), it was inversely linked to the fermentation, since it almost disappeared after $8 \mathrm{~h}$. Not all sourdoughs were characterised by the increase of benzaldehyde, as commonly reported for this kind of products (Chang, Seitz, \& Chambers, 1995; Seitz et al., 1998). The most noticeable differences among LAB were found for 3-methyl-1-butanol, ranging between 0.27 and 33.86, whose concentration is known to be dependent on the LAB strain performing the fermentation (Gobbetti et al., 1995).

Sourdough fermentation is essential to achieve an acceptable flavour; the comparison between chemically acidified bread and sourdough bread showed that the latter possessed a superior sensory quality (Kirchhoff \& Schieberle, 2002). However, the overall aroma profile of final bread, is due to the type of dominating LAB (Corsetti \& Settanni, 2007). Besides acetic acid, other compounds may play a defining role in the composition of VOCs. E.g. ethyl acetate content was higher in sourdoughs fermented with heterofermentative $\mathrm{LAB}$ compared to sourdoughs fermented with homofermentative $\mathrm{LAB}$, while an opposite trend was observed for the content of aldehydes that was higher in sourdoughs fermented with homofermentative cultures (Lund, Hansen, \& Lewis, 1989). However, due to evaporation during baking, the amounts of alcohols, esters and diacetyl in sourdough bread are much lower than in the corresponding sourdough (Hansen \& Hansen, 1996; Lund et al., 1989).

\subsection{Characterisation of breads}

After baking, the experimental breads were subjected to several determinations. In the experimentation carried out with sterile flour (Table 6), Ln. citreum PON10079 and PON10080 and all W. cibaria strains determined a final height of breads consistently higher than control bread, while in the productions performed with non-sterile flour, the height reached with $L b$. sanfranciscensis PON100100 was above those obtained with Ln. citreum and W. cibaria strains.

The colour of both the crust and crumb of control breads and those obtained with doughs inoculated with $\mathrm{LAB}$ with sterile or non-sterile flour were almost comparable, with only small differences registered for the parameters $a^{*}$ and $b^{*}$ of the crust for the sterile flour experimentation and $b^{*}$ of the crust for the non-sterile flour experimentation. Despite the fact that, in comparison with a non-inoculated dough the addition of LAB causes significant changes in the Hunter's scale parameters (García-Argueta et al., 2013), the colour of the final breads obtained in this study was not influenced by the different LAB strains added. However, all sourdoughs inoculated with $L A B$ were different from the control doughs.

The hardness was greatly influenced by LAB. In general, the softness of the final breads was directly correlated with the acidification kinetics, 
Table 6

Characteristics of experimental breads.

\begin{tabular}{|c|c|c|c|c|c|c|c|c|c|c|c|c|c|c|c|c|c|c|c|c|c|c|}
\hline \multirow[t]{2}{*}{ Strains } & \multirow{2}{*}{\multicolumn{2}{|c|}{$\begin{array}{l}\text { Height } \\
(\mathrm{mm})\end{array}$}} & \multicolumn{6}{|c|}{ Crust colour } & \multicolumn{6}{|c|}{ Crumb colour } & \multirow{2}{*}{\multicolumn{2}{|c|}{$\begin{array}{l}\text { Hardness } \\
(\mathrm{N})\end{array}$}} & \multirow{2}{*}{\multicolumn{2}{|c|}{$\begin{array}{l}\text { Void } \\
\text { fraction } \\
(\%)\end{array}$}} & \multirow{2}{*}{\multicolumn{2}{|c|}{$\begin{array}{l}\text { Cell density } \\
\left(\text { n. } \mathrm{cm}^{-2}\right)\end{array}$}} & \multirow{2}{*}{\multicolumn{2}{|c|}{$\begin{array}{l}\text { Mean } \\
\text { cell area } \\
\left(\mathrm{mm}^{2}\right)\end{array}$}} \\
\hline & & & $\mathrm{L}^{*}$ & & $a^{*}$ & & $b^{*}$ & & $\mathrm{~L}^{*}$ & & $a^{*}$ & & $b^{*}$ & & & & & & & & & \\
\hline \multicolumn{23}{|l|}{ Experimentation with sterile flour } \\
\hline control SFD & 26.00 & bc & 60.45 & $\mathrm{ab}$ & 1.62 & ad & 27.09 & ac & 57.70 & $a b$ & 0.20 & $\mathrm{a}$ & 19.75 & $\mathrm{a}$ & 26.53 & $\mathrm{a}$ & 18.58 & ef & 65.60 & $\mathrm{~cd}$ & 0.32 & $\mathrm{~cd}$ \\
\hline Lb. plantarum PON100274 & 27.60 & bc & 61.53 & $\mathrm{ab}$ & 1.16 & $\mathrm{~cd}$ & 24.29 & de & 55.82 & $a b$ & -0.49 & $a b$ & 17.39 & $\mathrm{~b}$ & 31.25 & a & 12.62 & f & 78.90 & bd & 0.10 & $f$ \\
\hline Lb. sanfranciscensis LMG $17498^{\mathrm{T}}$ & 28.00 & $\mathrm{~b}$ & 61.90 & $\mathrm{ab}$ & 1.49 & ad & 25.50 & ce & 61.69 & $a b$ & -0.09 & $a b$ & 18.25 & $a b$ & 15.18 & ce & 39.00 & $\mathrm{a}$ & 108.26 & $\mathrm{a}$ & 0.37 & Bd \\
\hline Lb. sanfranciscensis PON100100 & 28.30 & $\mathrm{~b}$ & 61.68 & $a b$ & 1.41 & ad & 25.48 & ce & 63.38 & $a b$ & -0.13 & $a b$ & 18.43 & $\mathrm{ab}$ & 17.13 & bc & 39.36 & $\mathrm{a}$ & 101.95 & $\mathrm{ab}$ & 0.42 & ad \\
\hline Lb. sanfranciscensis PON100336 & 31.30 & $a b$ & 62.01 & $a b$ & 1.61 & ad & 25.62 & be & 60.61 & $a b$ & 0.12 & $a b$ & 18.03 & $a b$ & 13.88 & $\mathrm{cf}$ & 38.57 & a & 111.70 & $\mathrm{a}$ & 0.35 & Bd \\
\hline Lb. sakei PON10098 & 22.00 & c & 58.57 & $\mathrm{~b}$ & 1.09 & d & 23.82 & e & 56.29 & $a b$ & -0.08 & $a b$ & 17.33 & $\mathrm{~b}$ & 20.63 & b & 22.09 & ce & 85.99 & ac & 0.26 & De \\
\hline Ln. citreum PON10021 & 26.00 & bc & 60.91 & $\mathrm{ab}$ & 1.29 & bd & 25.18 & ce & 54.27 & b & 0.06 & $a b$ & 18.25 & $\mathrm{ab}$ & 21.15 & b & 39.61 & $\mathrm{a}$ & 96.48 & $a b$ & 0.42 & Ad \\
\hline Ln. citreum PON10079 & 36.20 & $\mathrm{a}$ & 62.65 & $\mathrm{ab}$ & 1.88 & a & 26.91 & ac & 64.63 & $a b$ & -0.12 & $a b$ & 17.75 & $a b$ & 15.25 & $\mathrm{~cd}$ & 39.52 & $\mathrm{a}$ & 101.95 & $a b$ & 0.40 & Ad \\
\hline Ln. citreum PON10080 & 34.00 & $a b$ & 62.13 & $a b$ & 1.47 & ad & 26.17 & ae & 62.58 & $a b$ & -0.31 & $a b$ & 17.49 & $\mathrm{~b}$ & 11.48 & df & 27.91 & $\mathrm{~cd}$ & 78.46 & bd & 0.35 & $\mathrm{Bd}$ \\
\hline Ln. mesenteroides PON10031 & 32.00 & $a b$ & 63.02 & a & 1.76 & $\mathrm{ab}$ & 27.90 & $\mathrm{ab}$ & 62.68 & $\mathrm{ab}$ & -0.17 & $a b$ & 17.89 & $\mathrm{ab}$ & 13.35 & $\mathrm{cf}$ & 29.63 & bc & 57.62 & d & 0.51 & $\mathrm{Ab}$ \\
\hline Ln. pseudomesenteroides PON10024 & 29.00 & $\mathrm{~b}$ & 62.62 & $a b$ & 1.46 & ad & 26.30 & ad & 61.85 & $a b$ & -0.58 & $\mathrm{~b}$ & 17.51 & $\mathrm{~b}$ & 15.20 & ce & 21.44 & de & 87.32 & ac & 0.16 & Ef \\
\hline Ln. pseudomesenteroides PON100315 & 29.50 & $\mathrm{~b}$ & 60.84 & $a b$ & 1.29 & bd & 25.80 & be & 60.74 & $a b$ & -0.53 & $\mathrm{~b}$ & 16.51 & $\mathrm{~b}$ & 10.10 & f & 36.69 & $a b$ & 66.05 & $c d$ & 0.56 & A \\
\hline W. cibaria PON10030 & 36.00 & $\mathrm{a}$ & 63.37 & a & 1.30 & ad & 27.47 & $\mathrm{ac}$ & 62.89 & $\mathrm{ab}$ & -0.59 & $\mathrm{~b}$ & 17.56 & $\mathrm{~b}$ & 10.15 & ef & 40.25 & $\mathrm{a}$ & 90.43 & ac & 0.46 & Ac \\
\hline W. cibaria PON10032 & 34.20 & $a b$ & 63.39 & $\mathrm{a}$ & 1.81 & $\mathrm{ab}$ & 28.32 & $\mathrm{a}$ & 65.68 & $\mathrm{a}$ & -0.43 & $a b$ & 18.49 & $a b$ & 13.80 & $\mathrm{cf}$ & 42.50 & $\mathrm{a}$ & 93.31 & ac & 0.46 & Ac \\
\hline W. cibaria PON100337 & 35.80 & $\mathrm{a}$ & 62.39 & $a b$ & 1.69 & ac & 26.22 & ae & 62.10 & $a b$ & -0.26 & $a b$ & 17.26 & $\mathrm{~b}$ & 10.50 & df & 38.72 & $\mathrm{a}$ & 92.64 & ac & 0.43 & Ad \\
\hline SEM & 1.25 & & 0.21 & & 0.04 & & 0.18 & & 1.02 & & 0.07 & & 0.21 & & 1.48 & & 2.78 & & 6.18 & & 0.03 & \\
\hline Significance & $* *$ & & $* * *$ & & $* * *$ & & $* * *$ & & ** & & ** & & ** & & *** & & $* * *$ & & $* * *$ & & *** & \\
\hline \multicolumn{23}{|c|}{ Experimentation with non-sterile flour } \\
\hline control nSFD & 20.00 & c & 64.83 & bd & -0.19 & $\mathrm{ab}$ & 20.50 & de & 65.94 & $\mathrm{ab}$ & -1.33 & $a b$ & 15.96 & $\mathrm{a}$ & 29.38 & $\mathrm{a}$ & 20.77 & fg & 108.60 & ac & 0.19 & c \\
\hline control wnSFD & 20.00 & c & 63.87 & $\mathrm{~cd}$ & -0.34 & $\mathrm{~b}$ & 20.58 & ce & 63.81 & b & -1.36 & $a b$ & 16.11 & $\mathrm{a}$ & 28.43 & $a b$ & 15.58 & $\mathrm{~g}$ & 110.82 & $a b$ & 0.15 & c \\
\hline Lb. plantarum PON100274 & 24.00 & c & 67.53 & ac & -0.13 & $\mathrm{ab}$ & 19.59 & e & 68.64 & $a b$ & -1.37 & $a b$ & 15.66 & $\mathrm{ab}$ & 22.08 & ac & 14.85 & $\mathrm{~g}$ & 103.94 & ae & 0.14 & c \\
\hline Lb. sanfranciscensis LMG $17498^{\mathrm{T}}$ & 30.50 & $\mathrm{~b}$ & 68.32 & $\mathrm{ab}$ & 0.08 & $\mathrm{ab}$ & 22.76 & ac & 71.24 & $\mathrm{a}$ & -1.47 & $a b$ & 15.98 & $\mathrm{a}$ & 17.98 & bd & 37.52 & bc & 102.29 & ae & 0.38 & bc \\
\hline Lb. sanfranciscensis PON100100 & 36.00 & $\mathrm{a}$ & 68.66 & $\mathrm{a}$ & 0.46 & $\mathrm{a}$ & 23.53 & $a b$ & 69.42 & $a b$ & -1.42 & $a b$ & 14.34 & $a b$ & 15.23 & $\mathrm{~cd}$ & 36.43 & bc & 101.51 & ae & 0.36 & c \\
\hline Lb. sanfranciscensis PON100336 & 30.00 & $\mathrm{~b}$ & 68.36 & $a b$ & 0.00 & $\mathrm{ab}$ & 21.96 & be & 72.19 & a & -1.55 & $a b$ & 16.00 & $\mathrm{a}$ & 20.55 & ad & 39.44 & ac & 103.94 & ae & 0.39 & bc \\
\hline Lb. sakei PON10098 & 22.00 & c & 62.13 & d & 0.32 & $\mathrm{ab}$ & 20.57 & ce & 69.59 & $\mathrm{ab}$ & -1.22 & $\mathrm{a}$ & 14.23 & $a b$ & 20.48 & ad & 25.99 & $\mathrm{df}$ & 123.01 & $\mathrm{a}$ & 0.21 & c \\
\hline Ln. citreum PON10021 & 23.00 & c & 67.04 & ac & -0.24 & $\mathrm{ab}$ & 19.56 & e & 68.92 & $\mathrm{ab}$ & -1.28 & $\mathrm{a}$ & 14.88 & $a b$ & 27.13 & $\mathrm{ab}$ & 22.64 & eg & 127.88 & $\mathrm{a}$ & 0.18 & c \\
\hline Ln. citreum PON10079 & 32.00 & $a b$ & 68.36 & $a b$ & 0.05 & $\mathrm{ab}$ & 24.08 & $a b$ & 68.94 & $a b$ & -1.35 & $a b$ & 14.04 & $a b$ & 14.70 & $\mathrm{~cd}$ & 43.91 & $a b$ & 68.04 & be & 0.66 & $a b$ \\
\hline Ln. citreum PON10080 & 33.00 & $a b$ & 67.46 & ac & -0.27 & $\mathrm{~b}$ & 23.81 & $\mathrm{ab}$ & 68.49 & $\mathrm{ab}$ & -1.42 & $a b$ & 13.51 & $\mathrm{~b}$ & 11.90 & d & 32.79 & ce & 107.49 & ad & 0.31 & c \\
\hline Ln. mesenteroides PON10031 & 32.00 & $a b$ & 68.11 & $a b$ & -0.01 & $\mathrm{ab}$ & 22.53 & ac & 70.09 & $a b$ & -1.22 & $\mathrm{a}$ & 15.16 & $\mathrm{ab}$ & 16.43 & $\mathrm{~cd}$ & 48.80 & $\mathrm{a}$ & 67.15 & ce & 0.73 & $\mathrm{a}$ \\
\hline Ln. pseudomesenteroides PON10024 & 31.00 & $a b$ & 68.22 & $a b$ & 0.02 & $\mathrm{ab}$ & 23.42 & $\mathrm{ab}$ & 65.92 & $\mathrm{ab}$ & -1.48 & $a b$ & 14.73 & $a b$ & 13.95 & $\mathrm{~cd}$ & 35.01 & bd & 97.74 & ae & 0.36 & c \\
\hline Ln. pseudomesenteroides PON100315 & 30.00 & $\mathrm{~b}$ & 69.33 & $\mathrm{a}$ & -0.10 & $\mathrm{ab}$ & 23.00 & ac & 66.45 & $a b$ & -1.28 & $\mathrm{a}$ & 14.53 & $a b$ & 19.55 & bd & 38.55 & ac & 62.72 & d & 0.65 & $a b$ \\
\hline W. cibaria PON10030 & 30.00 & b & 68.62 & $a b$ & 0.08 & $\mathrm{ab}$ & 23.16 & ac & 70.92 & $\mathrm{a}$ & -1.32 & $a b$ & 14.87 & $\mathrm{ab}$ & 12.83 & $\mathrm{~cd}$ & 42.92 & ac & 64.72 & de & 0.68 & $\mathrm{a}$ \\
\hline W. cibaria PON10032 & 33.00 & $a b$ & 69.48 & $\mathrm{a}$ & 0.24 & $\mathrm{ab}$ & 23.00 & ac & 68.30 & $\mathrm{ab}$ & -1.68 & $\mathrm{~b}$ & 14.17 & $a b$ & 14.23 & $\mathrm{~cd}$ & 34.17 & bd & 112.81 & $\mathrm{a}$ & 0.30 & $c$ \\
\hline W. cibaria PON100337 & 33.00 & $a b$ & 69.26 & $\mathrm{a}$ & 0.00 & $\mathrm{ab}$ & 24.84 & $\mathrm{a}$ & 67.83 & $a b$ & -1.41 & $\mathrm{ab}$ & 14.68 & $a b$ & 16.55 & $\mathrm{~cd}$ & 37.75 & bc & 95.52 & ae & 0.40 & bc \\
\hline SEM & 1.44 & & 0.30 & & 0.04 & & 0.23 & & 0.41 & & 0.02 & & 0.16 & & 1.32 & & 2.32 & & 5.03 & & 0.05 & \\
\hline Significance & $* *$ & & $* * *$ & & $* * *$ & & $* * *$ & & $* * *$ & & $* *$ & & * & & $* * *$ & & $* * *$ & & $* * *$ & & $* * *$ & \\
\hline
\end{tabular}

Abbreviations: SFD, sterile flour dough; nSFD, non sterile flour dough; wnFDS, non sterile water non sterile flour; Lb., Lactobacillus; Ln., Leuconostoc; W., Weissella.

Results indicate mean values of four measurements (carried out in duplicate for two independent productions).

Data within a column followed by the same letter are not significantly different according to Tukey's test $\left(P\right.$ value: ${ }^{* * *}, P \leq 0.01 ;{ }^{* *}, P \leq 0.01 ; *, P \leq 0.05$; NS, not significant).

confirming that a soft crumb is associated with the acidification of the dough which reduces its elasticity and resistance to extension (Arendt, Ryan, \& Dal Bello, 2007; Clarke, Schober, Dockery, O’Sullivan, \& Arendt, 2004). The lowest values were reached by the strains W. cibaria PON10030 and PON100337, Ln. pseudomesenteroides PON100315 and Ln. citreum PON10080 with sterile flour. This observation was also made for $W$. cibaria PON10030 and Ln. citreum PON10080 in the presence of non-sterile flour. Compared to the control bread, a softer sourdough bread was obtained thanks to the action of $L$ n. citreum by Choi et al. (2012).

Recently, image analysis has been used as a quantitative tool for the assessment of crumb features (Farrera-Rebollo et al., 2012). In this study, the highest values of void fraction and cell density were registered for all W. cibaria strains, Ln. citreum PON10021 and PON10079 and Lb. sanfranciscensis PON100100 with sterile flour. In general, the values of void fraction and cell density displayed by the trials carried out with non-sterile flour were higher than those of the corresponding sterile flour trials. On average, cell density and mean cell area registered for the breads processed with the sterile flour showed lower values than those evaluated for the corresponding breads obtained with the non-sterile flour. Gonzales-Barron and Butler (2006) stated that slight variations in threshold led to substantial variations in crumb feature values, with cell uniformity and void fraction being more sensitive than the others. Thus, the differences estimated in this study for the different breads have to be considered consistent.

\section{Conclusions}

In this work, an integrated technological approach based on acidification, acid production, loaf height, colour formation, softness of bread and volatile compound generation applied on several flour LAB indicated the suitability of the strains Ln. citreum PON10079 and PON10080 and $W$. cibaria PON10030 and PON10032 to act as starter cultures for sourdough production.

\section{Acknowledgments}

This work was financially supported by the project for industrial research and training PON01_02249 "Application of molecular biotechnologies and pro-technological microorganisms for the characterisation and valorisation of dairy and bakery chains of typical products" of the Italian Ministry of Education, University and Research (CUP: B11C11000430005). The authors are grateful to the staff of the bakery "Piana delle Bontà" (Piana degli Albanesi, Italy) for their support during sourdough production and baking, to Dr. Antonella Paladino for her help with the microbiological 
analyses and Prof. Baldassare Portolano (University of Palermo) for his involvement in the realisation of the project. Mr Simon Gill is also thanked for proof-reading the final version in English.

\section{References}

Alfonzo, A., Ventimiglia, G., Corona, O., Di Gerlando, R., Gaglio, R., Francesca, N., et al. (2013). Diversity and technological potential of lactic acid bacteria of wheat flours. Food Microbiology, 36, 343-354.

Arendt, E. K., Ryan, L. A.M., \& Dal Bello, F. (2007). Impact of sourdough on the texture of bread. Food Microbiology, 24, 165-174

Chang, C. Y., Seitz, L. M., \& Chambers, E. (1995). Volatile flavor components of breads made from hard red winter wheat and hard white winter wheat. Cereal Chemistry, 72, 237-242.

Choi, H., Kim, Y. -W., Hwang, I., Kim, J., \& Yoon, S. (2012). Evaluation of Leuconostoc citreum HO12 and Weissella koreensis HO20 isolated from kimchi as a starter culture for whole wheat sourdough. Food Chemistry, 134, 2208-2216.

Clarke, C. I., Schober, T., Dockery, P., O'Sullivan, K., \& Arendt, E. K. (2004). Wheat sourdough fermentation: Effects of time and acidification on fundamental rheological properties. Cereal Chemistry, 81, 409-417.

Corsetti, A., \& Settanni, L. (2007). Lactobacilli in sourdough fermentation: A review. Food Research International, 40, 539-558.

Corsetti, A., Settanni, L., Chaves-López, C., Felis, G. E., Mastrangelo, M., \& Suzzi, G. (2007b). A taxonomic survey of lactic acid bacteria isolated from wheat (Triticum durum) kernels and non-conventional flours. Systematic and Applied Microbiology, 30, 561-571.

Corsetti, A., Settanni, L., Valmorri, S., Mastrangelo, M., \& Suzzi, G. (2007a). Identification of subdominant sourdough lactic acid bacteria and their evolution during laboratory-scale fermentations. Food Microbiology, 24, 592-600.

Czerny, M., \& Schieberle, P. (2002). Important aroma compounds in freshly ground wholemeal and white wheat flour-identification and quantitative changes during fermentation. Journal of Agricultural and Food Chemistry, 50, 6835-6840.

Ehrmann, M.A., \& Vogel, R. F. (2005). Molecular taxonomy and genetics of sourdough lactic acid bacteria. Trends in Food Science and Technology, 16, 31-42.

Farrera-Rebollo, R. R., de la Paz Salgado-Cruz, M., Chanona-Pérez, J., Gutiérrez-López, G. F., Alamilla-Beltrán, L., \& Calderón-Domínguez, G. (2012). Evaluation of image analysis tools for characterization of sweet bread crumb structure. Food and Bioprocess Technology, 5, 474-484.

García-Argueta, I., Dublán-García, O., Quintero-Salazar, B., Dominguez-Lopez, A., Gómez-Oliván, L. M., \& Salem, A. -F. Z. M. (2013). Effect of lactic acid bacteria on the textural properties of an edible film based on whey, inulin and gelatin. African Journal of Biotechnology, 19, 2659-2669.

Gobbetti, M., Simonetti, M. S., Corsetti, A., Santinelli, F., Rossi, J., \& Damiani, P. (1995). Volatile compound and organic acid production by mixed wheat sour dough starters: Influence of fermentation parameters and dynamics during baking. Food Microbiology, 12, 497-507

Gonzales-Barron, U., \& Butler, F. (2006). A comparison of seven thresholding techniques with the k-means clustering algorithm for measurement of bread-crumb features by digital image analysis. Journal of Food Engineering, 74, 268-278.

Hammes, W. P., \& Gänzle, M. G. (1998). Sourdough breads and related products. In B. J. B. Woods (Ed.), Microbiology of fermented foods, Vol. 1. (pp. 199-216)London: Blackie Academic \& Professional.

Hammes, W. P., \& Vogel, R. F. (1995). The genus Lactobacillus. In B. J. B. Wood, \& W. H. Holzapfel (Eds.), The genera of lactic acid bacteria (pp. 19-54). London: Blackie Academic \& Professional.

Hansen, A., \& Hansen, B. (1996). Flavour of sourdough wheat bread crumb. Zeitschrift für Lebensmittel-Untersuchung und -Forschung, 202, 244-249.
Hansen, A., \& Lund, B. (1987). Volatile compounds in rye sourdough. In M. Martens, G. A. Dalen, \& H. Russwurm (Eds.), Flavour science and technology (pp. 43-49). New York: Wiley.

Kirchhoff, E., \& Schieberle, P. (2002). Quantitation of odor-active compounds in rye flour and rye sourdough using a stable isotope dilution assay. Journal of Agricultural and Food Chemistry, 50, 5311-5378.

Kline, L., \& Sugihara, T. F. (1971). Microorganisms of the San Francisco sour dough bread process. II. Isolation and characterization of undescribed bacterial species responsible for the souring activity. Applied Microbiology, 21, 459-465.

Lorenz, K. (1983). Sourdough processes. Methodology and biochemistry. Baker's Digest $55,85-91$.

Lund, B., Hansen, A., \& Lewis, M. J. (1989). The influence of dough yield on acidification and production of volatiles in sour doughs. Lebensmittel Wissenschaft und Technologie, 22, $150-153$.

Meignen, M., Onno, B., Gelinas, B. P., Infantes, M., Guilois, S., \& Cahagnier, B. (2001). Optimization of sourdough fermentation with Lactobacillus brevis and baker's yeast. Food Microbiology, 18, 239-245.

Minervini, F., De Angelis, M., Di Cagno, R., Pinto, D., Siragusa, S., Rizzello, C. G., et al. (2010). Robustness of Lactobacillus plantarum starters during daily propagation of wheat flour sourdough type I. Food Microbiology, 27, 897-908.

Minervini, F., Lattanzi, A., De Angelis, M., Di Cagno, R., \& Gobbetti, M. (2012). Influence of artisan bakery- or laboratory-propagated sourdoughs on the diversity of lactic acid bacterium and yeast microbiotas. Applied and Environmental Microbiology, 78 5328-5340.

Moroni, A. V., Arendt, E. K., \& Dal Bello, F. (2001). Biodiversity of lactic acid bacteria and yeasts in spontaneously-fermented buckwheat and teff sourdoughs. Food Microbiology, 28, 497-502.

Plessas, S., Fisher, A., Koureta, K., Psarianos, C., Nigam, P., \& Koutinas, A. A. (2008) Application of Kluyveromyces marxianus, Lactobacillus delbrueckii ssp. bulgaricus and L. helveticus for sourdough bread making. Food Chemistry, 106, 985-990.

Plessas, S., Trantallidi, M., Bekatorou, A., Kanellaki, M., Nigam, P., \& Koutinas, A. A. (2007) Immobilization of kefir and Lactobacillus casei on brewery spent grains for use in sourdough wheat bread making. Food Chemistry, 105, 187-194.

Robert, H., Gabriel, V., \& Fontagné-Faucher, C. (2009). Biodiversity of lactic acid bacteria in French wheat sourdough as determined by molecular characterization using species-specific PCR. International Journal of Food Microbiology, 135, 53-59.

Rosenquist, H., \& Hansen, A. (1998). The antimicrobial effect of organic acids, sour dough and nisin against Bacillus subtilis and B. licheniformis isolated from wheat bread. Journal of Applied Microbiology, 85, 621-631.

Schober, T. J., Messerschmidt, M., Bean, S. R., Park, S. H., \& Arendt, E. K. (2005). Glutenfree bread sorghum: Quality difference among hybrids. Cereal Chemistry, 82, 394-404.

Seitz, L. M., Chung, O. K., \& Rengarajan, R. (1998). Volatiles in selected commercial breads Cereal Chemistry, 75, 847-853.

Settanni, L., Massitti, O., Van Sinderen, D., \& Corsetti, A. (2005). In situ activity of a bacteriocin-producing Lactococcus lactis strain. Influence on the interactions between lactic acid bacteria during sourdough fermentation. Journal of Applied Microbiology, 99, 670-681.

Settanni, L., Miceli, A., Francesca, N., \& Moschetti, G. (2012). Investigation of the hygienic safety of aromatic plants cultivated in soil contaminated with Listeria monocytogenes. Food Control, 26, 213-219.

Spicher, G. (1983). Baked goods. In H. J. Rehm, \& G. Reed (Eds.), Biotechnology (pp. 1-80). Weinheim: Verlag Chemie.

Valmorri, S., Tofalo, R., Settanni, L., Corsetti, A., \& Suzzi, G. (2010). Yeast microbiota associated with spontaneous fermentation of traditional wheat sourdough breads of Abruzzo region (Italy). Antonie Van Leeuwenhoek International Journal of General and Molecular Microbiology, 97, 119-129.

Vogel, R. F., Knorr, R, Müller, M. R. A., Stendel, U. Gänzle, M. G. \& Ehmann, M.A. (1999) Non-dairy lactic fermentation: The cereal world. Antonie Van Leeuwenhoek International Journal of General and Molecular Microbiology, 76, 403-411. 